\title{
Arsenic, syphilis, and cancer of the prostate*
}

\author{
R E M LEES, R STEELE, AND D WARDLE
}

From the Department of Community Health and Epidemiology, Queen's University, Kingston K7L 2N6, Ontario, Canada

SUMMARY A study was conducted to investigate the association of cancer of the prostate with historical records of previous venereal disease and treatment with arsenical drugs. Eighty-three cancer cases were each age matched with two male controls-one with benign prostatic hypertrophy and one other non-cancer patient. Analysis from pooled data showed a statistically significant difference for syphilis infection between cases and controls at $p<0.05$; odds ratio 2.9. Matched pair analysis maintained $p<0.05$ significance for the first control group but odds ratios were 5.5 and 2.75 in respect of control groups 1 and 2 . The mean age at which venereal infection was acquired was higher for syphilis than for gonorrhoea, but the mean age at diagnosis of cancer was younger for the syphilis group; there was a statistically significant difference between the mean at cancer diagnosis of those who had syphilis and those who had no record of previous venereal infection $(t=2 \cdot 18$, df 70, p<0.05). No association was demonstrated for history of treatment with arsenical drugs.

Since the first case-control study of the relation of sexual characteristics and prostate cancer was reported by Steele et al in $1971^{1}$ other studies have confirmed the findings. Krain confirmed the greater number of sexual partners and higher coital frequency experienced by cancer of the prostate patients compared with their controls. Both Krain ${ }^{2}$ and Jackson $e a^{3}$ found a higher proportion of cases than controls with histories of venereal disease.

It was deduced that sexual activity was a reflection of hormonal activity and the increased frequency of venereal disease a consequence of greater sexual drive among the cases. Armerian et $\mathrm{al}^{4}$ assumed an underlying genetic susceptibility among cases in hypothesising a hormonal model for prostatic cancer, the genetic factor predisposing to higher fertility and prostatic cancer. That hypothesis would also explain the familial history of the disease which has been recorded by Woolf, ${ }^{5}$ Lynch, ${ }^{6}$ and others.

A viral aetiology of prostatic cancer would be consistent with the findings of multiple sexual partners and increased incidence of venereal disease among cases which have been reported by these

*This paper was presented at the IEA Xth Scientific Meeting 1984 in Vancouver, Canada. authors. Herpes virus, Simian virus 40 , and cytomegalo virus have been studied and recovered from the male genital tract; there have been similar findings in experimental animals. ${ }^{7-9}$ Herpes virus 2 has been considered a possible aetiological agent in cancer of the uterine cervix. ${ }^{10}$ If that is correct and if herpes virus 2 is also involved in the development of prostatic cancer, one would expect a higher incidence than usual of cervical cancer in the wives of prostatic cancer patients, and vice versa. Lees and Steele ${ }^{11}$ used record linkage techniques to explore the possibility of such an association between 412 Saskatchewan women who died from cancer of the cervix and their husbands. They also assessed, by direct interview, the incidence of cancer of the cervix in the wives of prostatic cancer patients. In neither investigation could they find evidence to support the hypothesis.

In a previous study during a clear-out of old hospital records in Kingston, Ontario, we were able to examine Venereal Disease Clinic files for the late 1930 s, 1940s, and 1950s. With the aid of the Ontario Cancer Treatment and Research Foundation 71 cancer deaths from among 1093 clinic records were identified. Four patients had died from cancer of the prostate and all had been treated with arsenic at the 
VD Clinic. Record linkage was obviously incomplete and at best could be attempted only for persons who had died in Ontario. The results, however, permitted speculation on the carcinogenicity of arsenic in relation to the prostate and the possibility of an association between infection with syphilis and subsequent cancer of the prostate.

\section{Method}

The objectives of the present study were to identify any association between cancer of the prostate and $(a)$ a past history of venereal infection or $(b)$ previous treatment with parenteral arsenical drugs. A case-control design was used for the study.

All patients treated for prostatic cancer at the National Defence Medical Center (NDMC) between 1962 and 1982 were identified from the hospital records. Each was matched by age at the time of treatment with two control subjects, one who had been treated at NDMC for benign prostatic hypertrophy (group 1) and another who had been treated for medical or surgical non-cancerous conditions (group 2). These control groups were similar to those used by the authors in the 1971 study. Cancer cases were accepted only after corroboration of the histological diagnosis. The age at diagnosis, blood group, height, weight, blood pressure, marital status, smoking history, and alcohol consumption were noted.

Permission was obtained to review the records of the NDMC in Ottawa, giving adequate safeguards to the maintenance of confidentiality. The military service records of past members of the armed forces are stored in the National Archives, and permission to examine these was also obtained. Thus documented information on part of the past medical history of the selected study subjects was available, for example, age at enlistment, whether career or war-time service, job category, entry and discharge ranks, diagnoses and dates of venereal infections, treatment received for venereal infection, and age at time of infection. No patient was interviewed; no information was obtained from individual recall, and all of the data abstracted had been recorded objectively by a third party.

The data were coded and entered into an IBM computer. Analyses were made using the Statistical Analysis System (SAS) program.

\section{Results}

A total of 83 cases of prostatic cancer met the entry criteria. The mean age of the cases was 69.9 years (SD 10.0), median $71 \cdot 0$ years, and matching with two controls was possible to within 1 year of age.
The distribution of military ranks between the cases and control groups was similar.

The distribution of venereal diseases by recorded diagnoses for each of the groups is shown in table 1. The difference in frequency of venereal disease history between cases and controls combined or singly was not significant.

Table 1 Analysis of pooled data

\begin{tabular}{|c|c|c|c|c|c|c|}
\hline & \multicolumn{2}{|c|}{$\begin{array}{l}\text { Cases } \\
(N=83)\end{array}$} & \multicolumn{2}{|c|}{$\begin{array}{l}\text { Control I } \\
(N=83)\end{array}$} & \multicolumn{2}{|c|}{$\begin{array}{l}\text { Control } 2 \\
(N=83)\end{array}$} \\
\hline & $N$ & $\begin{array}{l}\text { OR } \\
\text { (All controls) }\end{array}$ & $N$ & $O R$ & $N$ & $O R$ \\
\hline No venereal disease & 56 & & 68 & & 60 & \\
\hline Gonorrhoea & 13 & $(0 \cdot 8)$ & 13 & & 17 & \\
\hline Syphilis & 12 & $(2 \cdot 9)$ & 4 & $(3 \cdot 3)$ & 5 & $(2 \cdot 6)$ \\
\hline Type not confirmed & 4 & & 0 & & 2 & \\
\hline All venereal disease & 29 & $(1 \cdot 6)$ & 17 & & 24 & \\
\hline
\end{tabular}

$\mathrm{OR}=$ odds ratio cases.

There was a significant difference for past history of syphilis between cases and the combined control groups, $x^{2}=6.44, p<0.05$.

Significance of difference for control group $1, \chi^{2}=4 \cdot 89, p<0 \cdot 05$, for control group $2, \chi^{2}=3.42$ just short of $p<0.05$.

Four cases of venereal disease in the cases group and two in the control group were not labelled diagnostically as syphilis or gonorrhoea because of inadequate records: laboratory confirmation was not available. In the cases group, however, clinical notes suggest that two were syphilis and two gonorrhoea while there would be one in each diagnostic category from the doubtful controls.

For calculation of statistical significance of differences between groups for specific venereal diseases the cases whose type of infection was in doubt were excluded. Syphilis was found to be significantly associated with subsequent development of cancer of the prostate compared to the combined controls; gonorrhoea was not.

The relative risks of developing cancer of the prostate for cases versus combined and individual groups are also shown in the table.

The results of matched pair analysis are provided in table 2 .

Table 2 History of venereal disease by type: matched pair analysis

\begin{tabular}{ll}
\hline Cases $v$ control group 1 for history of syphilis & $x^{2}=4.92$ 1DF $p<0.05$ \\
Cases $v$ control group 2 for history of syphilis & $\chi^{2}=2.401 \mathrm{DF} p<0.2$ \\
Cases $v$ control group 1 for history of gonorrhoea & $\mathrm{OR}=2.75$ \\
Cases $v$ control group 2 for history of gonnorhoea & $\mathrm{OR}=1$ \\
& $\mathrm{NS}=0.72$ \\
\hline
\end{tabular}

Method: Mandel, J. 
Further analysis of matched data, using both control groups, was performed, and the results are shown in Table 3.

Table 4 shows the mean age at diagnosis of the diseases being considered. The mean age at diagnosis of cancer was significantly different for those who had a history of syphilis and for those who had had no venereal disease. Gonorrhoea had been acquired at an earlier mean age than syphilis in both cases and control groups.

Table 3 History of syphilis: matched data analysis using both control groups

\begin{tabular}{lllll}
\hline & \multicolumn{4}{l}{ Number controls positive } \\
\cline { 2 - 5 } & 0 & 1 & 2 & Total \\
\hline Case positive & 10 & 2 & 0 & 12 \\
Case negative & 59 & 6 & 0 & 65 \\
Totals & 69 & 8 & 0 & 77 \\
\hline
\end{tabular}

$\chi^{2}=5 \cdot 84$

DF 2

$\mathrm{p}<0.1>0.05$

$\mathrm{OR}=3.66$

Method: Breslow and Day

Table 4 Mean age (years) at diagnosis

\begin{tabular}{lll}
\hline & Cases & Controls \\
\hline Gonorrhoea & $26 \cdot 3$ & $26 \cdot 5$ \\
Syphilis & $29 \cdot 9$ & $32 \cdot 5$ \\
Cancer (gonorrhoea group) & $68 \cdot 2$ & - \\
Cancer (syphilis group) & $64 \cdot 6^{*}$ & - \\
Cancer (no VD group) & $71 \cdot 0^{*}$ & - \\
\hline
\end{tabular}

*Difference in mean age between the syphilis group and the no VD group. $\mathrm{t}=2 \cdot 18$

DF 70

$\mathrm{p}<0.05$

Among the cases there was a difference in the latent period between acquisition of the two types of veneral disease and the diagnosis of cancer-a mean of 33.7 years for syphilis patients and 40.6 years for gonorrhoea patients. This difference did not reach statistical significance $(t=1 \cdot 44$, df $21, p>0 \cdot 1)$.

Twenty men, 5 cases and 15 controls, had a documented record of treatment with parenteral arsenical drugs. Five cases and five controls had been treated with arsenicals for syphilis. The statistical analyses of difference between the groups with respect to arsenical treatment is illustrated in table 5 . There were no significant differences between cases and controls for history of exposure to arsenical drugs.

Dates of birth of the subjects studied ranged from 1886 to 1925 (median 1909), and infections with syphilis occurred from 1917 to 1961 .
Table 5 Differences in arsenical treatment

\begin{tabular}{lcc}
\hline & \multicolumn{2}{l}{ All arsenical treatment } \\
\cline { 2 - 3 } & $\begin{array}{l}\text { Cases } \\
(N=83)\end{array}$ & $\begin{array}{l}\text { Controls } \\
(N=166)\end{array}$ \\
\hline Arsenicals used & 5 & 15 \\
No arsenicals used & 78 & 151 \\
\hline$\chi^{2}=0.66$ & & \\
$0 \cdot 5>p>0 \cdot 1$ & & \\
NS & & \\
\hline
\end{tabular}

\begin{tabular}{lc} 
Arsenical treatment for syphilis \\
\hline Cases \\
$(N=12)$ & $\begin{array}{l}\text { Controls } \\
(N=9)\end{array}$ \\
\hline 5 & 5 \\
7 & 4
\end{tabular}

$x^{2}=0.036$

NS

The case and control groups were examined for a possible cohort effect and none was found. The subjects had been closely age matched but there was no obvious difference in occupation, rank or theatre of operations.

\section{Discussion}

Military medical records may not be complete, particularly in peace time when there may be more likelihood of men attending civilian doctors. However, we have no reason to believe that there were differences in rank or location of service between the cases and control groups which would result in more inaccuracy in one group than in the others. It is likely that the accuracy or completeness of military records is greater than that of individual patient recall or past history as recorded on hospital patient records. It must also be remembered that the documentation of illness and treatment covered only the period of the men's service in the armed forces.

This study did not demonstrate, as others have, a difference in the incidence of undifferentiated venereal diseases between the cases of prostatic cancer and their controls. It did show that, while there were no differences between the groups for all venereal disease or gonorrhoea, there was a significant difference at the $\mathrm{p}<0.05$ level in the incidence of syphilis when the data were analysed with the control groups combined. When analysis was made with the control groups separated the difference between cases and control group 1 (benign prostatic hypertrophy cases) remained at the $\mathrm{p}<0.05$ level; with control group 2 the difference was just short of significance at the $p=0.05$ level. One must consider the real meaning of chance at 1 in 19 as 
opposed to 1 in 20 , the latter being a somewhat arbitrary cut point of significance.

Matched pair analysis provided a similar result for control group 1 , with an odds ratio of $5 \cdot 5$ for cases, but although the significance of difference dropped in the second control group the odds ratio was $2 \cdot 75$.

Matched data analysis using both control groups showed a difference between cases and controls which was again just short of the $p<0.05$ level, but the odds ratio was $3 \cdot 66$.

From the relative risk estimates and the closeness of all significances of difference to $0<0 \cdot 05$, one must deduce that the association between acquired syphilis and cancer of the prostate is probably real.

The possibility of an association with syphilis was strengthened by the observation that cases with a past history of syphilis had the diagnosis of prostatic cancer made at an earlier age than those with a history of gonorrhoea or those without a history of venereal disease of any type. This difference in age was statistically significant for syphilis but not for gonorrhoea.

Men who had had gonorrhoea acquired the infection at a younger mean age than those who had syphilis. The mean latent period between syphilis infection and the onset of cancer of the prostate was shorter than the mean latent period for gonorrhoea victims -33.7 years versus $40 \cdot 6$ years.

These age and latency period differences suggest exposure to a more active carcinogenic stimulus or to a higher dose of the carcinogen. The carcinogen does not appear to be related to earlier sexual intercourse or coitus with multiple partners per se but rather to the acquisition of a specific infection or some other factor associated with that infection. We could not find evidence that that other factor was treatment with arsenical drugs.

There have been several suggestions, as the result of previous studies, that the higher frequency of venereal disease history among prostatic cancer victims reflects a more intense sexual drive and thus supports the hypothesis that male hormones are somehow carcinogenic for the prostate. While this study does not refute that hypothesis or gainsay the previously found associations between the disease and sexual drive, it does suggest that syphilis had a more specific association with the subsequent development of prostatic cancer than does venereal disease in general. Syphilitic glossitis leads to the development of leukoplakia, which frequently becomes malignant. ${ }^{12}$ The Treponema pallidum might in certain circumstances be carcinogenic for the prostate. There was not evidence that the gonococcus had a similar role.

Syphilis is obviously not a necessary precursor of prostatic cancer, but a past history of syphilis having an odds ratio of 2.75 to 5.5 in separate control groups, further investigation of the nature of the association is warranted.

This study was supported by a grant from Health and Welfare, Canada.

We would like to record our appreciation of the assistance given by the Surgeon General and the staff of the National Defence Medical Center in Ottowa.

The help given by the National Archives of Canada is also gratefully acknowledged.

\section{References}

${ }^{1}$ Steele R, Lees REM, Kraus AS, Rao C. Sexual factors in the epidemiology of cancer of the prostate. J Chron Dis 1971; 24: 29-37.

${ }^{2}$ Krain LS. Epidemiologic variables in prostatic cancer. Geriatrics, 1973; 28: 93-8.

${ }^{3}$ Jackson MA, Aluwalia BS, Attah EB, Connelly CA, et al. Characteristics of prostatic cancer among blacks: a preliminary report. Cancer Chemother 1975; 59, 3-15.

${ }^{4}$ ArmerianHK, Lilienfeld AM, Diamond EL, Bross ID. Epidemiologic characteristics of patients with prostatic neoplasms. Am J Epidemiol 1975; 102, 47-54.

${ }^{5}$ Woolf GM. An investigation of the familial aspects of cancer of the prostate. Cancer 1960; 13: 739-43.

${ }^{6}$ Lynch HT, Larsen AL, Magnusson CW, Krush AJ. Prostatic carcinoma and multiple primary malignancies. Science 1972; 178, 318-20.

${ }^{7}$ Centifanto YM, Drylie DM, Deardonoff SL, Kaufman E. Herpesvirus type 2 in the male genital tract. Science 1972; 178: 318-20.

${ }^{8}$ Centifanto YM, Kaufman HE, Zam FS, Drylie DM, et al. Herpesvirus particles in prostatic carcionoma cells. $J$ Virol 1973; 12, 1608-11.

${ }^{9}$ Sanford EJ, Geder L, Laychock A, Rohnier TJ, et al. Evidence for the Association of cytomegalovirus with carcionoma of the prostate. J Urol 1977; 118: 789-92.

${ }^{10}$ Rawls WE, Tompkins WAF, Figuerao ME, Melnick JL. Herpesvirus 2: association with carcinoma of the cervix. Science 1968; 161, 1255-6.

${ }^{11}$ Lees REM, Steele R. Attempts to trace the incidence of cancer of the cervix in the spouses of patients with cancer of the prostate. Annual Report of the Ontario Cancer Treatment and Research Foundation 1971; 142-7.

${ }^{12}$ Erich JB. Diseases of the mouth. In Textbook of medicine, 10th ed., 1060. Eds Cecil RL, Loeb RF. Philadelphia and London: Saunders and Co. 